INPLASY

PROTOCOL

To cite: Yang et al. Risk factors of personal protective equipment-related pressure injury in medical staff: a Metaanalysis. Inplasy protocol 202210049. doi:

10.37766/inplasy2022.1.0049

Received: 10 January 2022

Published: 10 January 2022

Corresponding author:

Pengyu Yang

446878186@qq.com

Author Affiliation:

Hainanyixueyuan

Support: isrpfgsohmc

(HYYS2020-21).

Review Stage at time of this submission: Preliminary searches.

Conflicts of interest:

None declared.

\section{Risk factors of personal protective equipment-related pressure injury in medical staff: a Meta-analysis}

Review question / Objective: With the high incidence of DRPI in medical staff, many scholars have carried out research on the occurrence and influencing factors of DRPI in medical staff, but the research conclusions on the risk factors of DRPI in medical staff are different. This method was used to identify the risk factors of DRPI in medical staff, and to provide evidence for early clinical prevention of DRPI. p:medical staff I:personal protective equipment o:pressure injury S:Crosssectional, cohort, and case-control studies.

Information sources: Cochrane Library, PubMed, Web Of Science, CNKI database, Wanfang database, VIP database and China Biomedical Literature Database.

INPLASY registration number: This protocol was registered with the International Platform of Registered Systematic Review and Meta-Analysis Protocols (INPLASY) on 10 January 2022 and was last updated on 10 January 2022 (registration number INPLASY202210049).

\section{INTRODUCTION}

Review question / Objective: With the high incidence of DRPI in medical staff, many scholars have carried out research on the occurrence and influencing factors of DRPI in medical staff, but the research conclusions on the risk factors of DRPI in medical staff are different. This method was used to identify the risk factors of DRPI in medical staff, and to provide evidence for early clinical prevention of DRPI. p:medical staff I:personal protective equipment 0 :pressure injury $\mathrm{S}$ :Crosssectional, cohort, and case-control studies.

Condition being studied: As of now, the global infection rate and death rate have exceeded 230 million and more than 4 million, respectively, of which more than 120,000 have been diagnosed with CoVID-19 in China. Since COVID-19 is 
mainly transmitted through respiratory droplets and there is aerosol transmission, the National Health Commission recommends that all personnel in medical institutions who come into contact with suspected or confirmed COVID-19 patients must wear personal protective equipment (PPE), including Masks, gloves, goggles, protective clothing, etc. In the early days of COVID-19, considering the shortage of materials and human resources, medical staff often had to work continuously for 8-12 hours. With the prolongation of wearing time, PPE caused a large number of medical staff to suffer from pressure injuries of varying degrees and even facial skin. Collapse, known as device-related pressure injury (device-related pressure injury, DRPI). Studies have shown that the prevalence of DRPI in medical staff is as high as $\mathbf{3 0 . 0 3 \%}$. At present, many scholars have carried out research on the occurrence and influencing factors of DRPI in medical staff, but the research conclusions on the risk factors of DRPI in medical staff are different.

\section{METHODS}

Search strategy: search term: (PPE / Personal Protective Equipment/protective suit/masks/coverall/glove/shoe cover/ Goggles/ protective masks) AND (pressure damage/scar/medical device-related pressure ulcers/MDRPU/Pressure Injuries/ Pressure ulcer/device-related pressure injury/DRPI) AND (risk factors/ influence factors / related factors / dangerous factors) Databases: Cochrane Library, PubMed, Web Of Science, CNKI database, Wanfang database, VIP database and China Biomedical Literature Database.

Participant or population: Medical staff.

Intervention: Personal protective equipment.

Comparator: Pressure injury.

Study designs to be included: Crosssectional, cohort, and case-control studies.
Eligibility criteria: Inclusion criteria: (1)The research subjects were medical staff using PPE, aged $\geq 18$ years; (2)The research content was the risk factors of DRPI; (3)The research type was cross-sectional study, cohort study, and case-control study; (4)The diagnostic criteria for DRPI injury were clear; (5)Speech Limited to Chinese and English.Exclusion criteria: 1. Repeated publication; 2. Studies with data that cannot be extracted, no full text or incomplete information; 3. Studies that do not fit the theme.

Information sources: Cochrane Library, PubMed, Web Of Science, CNKI database, Wanfang database, VIP database and China Biomedical Literature Database.

Main outcome(s): Factors affecting pressure ulcers.

Additional outcome(s): Factors affecting pressure ulcers.

Data management: Endnote,Literature search, screening and information extraction were independently completed by two postgraduates according to the inclusion and exclusion criteria of the literature.

Quality assessment / Risk of bias analysis: Newcastle-Ottawa Scale, NOS.

Strategy of data synthesis: For enumeration data, odds ratio (OR) and $95 \%$ $\mathrm{CI}$ were selected, and continuous variable data were expressed by mean difference (MD) and $95 \% \mathrm{Cl}$. If the heterogeneity test $P>0.1,12<50 \%$, it means that there is homogeneity among the studies, and the fixed effect model is used; if $P \leq 0.1,12 \geq 50 \%$, it indicates that the heterogeneity among the studies is large, and the selection sensitivity Analysis or subgroup analysis was performed to find the source of heterogeneity. If the heterogeneity of the results was still large, the random effect model was used to calculate the combined amount or the combination was abandoned and descriptive analysis was used. 
Subgroup analysis: Subgroup studies were conducted according to factors such as PPE type, wearing time, and gender of medical staff.

Sensitivity analysis: After deleting any one of the documents, the combined results of the remaining documents are not much different from those when they were not deleted, which means that the sensitivity analysis has passed.

Country(ies) involved: China.

Keywords: medical personnel; personal protective equipment; device-related pressure injuries; risk factors.

Contributions of each author:

Author 1 - Pengyu Yang.

Author 2 - Hua Zhang.

Author 3 - Na Zhang. 